\title{
Design and commissioning of an ion guide system for In-Gas Laser Ionization and Spectroscopy experiments
}

\author{
S. Sels ${ }^{\mathrm{a}, 1, *}$, R. Ferrer ${ }^{\mathrm{a}}$, K. Dockx ${ }^{\mathrm{a}}$, C. Granados Buitrago ${ }^{\mathrm{a}, 1}$, M. Huyse $^{\mathrm{a}}$, Yu. Kudryavtsev ${ }^{\mathrm{a}}$, S. Kraemer $^{\mathrm{a}}$, S. Raeder $^{\mathrm{a}, 2}$, \\ P. Van Den Bergh ${ }^{\mathrm{a}}$, P. Van Duppen ${ }^{\mathrm{a}}$, M. Verlinde ${ }^{\mathrm{a}}$, E. Verstraelen ${ }^{\mathrm{a}}$, A. Zadvornaya ${ }^{\mathrm{a}}$ \\ ${ }^{a}$ KU Leuven, Instituut voor Kern- en Stralingsfyisca, B-3001 Leuven, Belgium
}

\begin{abstract}
Radio-frequency (RF) ion guides, also known as Linear Paul Traps, are powerful devices to efficiently transport ion beams from high to low pressure regions while keeping good ion optical properties. A set of ion guides comprising three different RF quadrupole (RFQ) structures has been designed using ion-trajectory simulations to improve the performance of the In-Gas Laser Ionizations and Spectroscopy (IGLIS) technique currently under development at KU Leuven. Results of the commissioning tests for the total transport efficiency and transient time through the ion guides as well as the longitudinal energy spread and transverse emittance are found to be in agreement with ion trajectory simulations considering a realistic ion-atom interaction potential.
\end{abstract}

\section{Introduction}

Laser spectroscopy is a powerful technique to experimentally determine ground- and isomeric-state properties of radioactive isotopes such as nuclear spins, electro5 magnetic moments and differences in nuclear mean-square charge radii $\delta\left\langle r^{2}\right\rangle[1,2]$. While collinear laser spectroscopy provides a high spectroscopic resolution [3], in-source ionization spectroscopy is more efficient. Using the latter technique, isotopes with production rates down to 0.1 ions $/ \mathrm{s}$ 10 can be measured [4-8]. However, due to Doppler and pressure broadening, in-source techniques only provide spectral resolutions in the order of a few GHz. This limits their applicability to isotopes with large hyperfine splittings (HFS) and isotope shifts (IS). More specifically, medium to heavy-mass nuclei are often studied using in-source techniques due to the increasing contribution of the Field shift $\left(\propto \delta\left\langle r^{2}\right\rangle\right)$ to the total IS [1]. Determining ground-state properties of the heavy elements is mandatory to further test, benchmark and develop state-of-the-art nuclear mod-

20 els. Because of the low production rates of these elements, experiments in this region of the nuclear chart are very challenging and data is scarce [2].

Using the In-Gas Laser Ionization and Spectroscopy 25 (IGLIS) technique, radioactive ions are thermalized and neutralized in a high-pressure gas cell (filled with $\approx 300 \mathrm{mbar}^{35}$ argon gas) at room temperature, like the one presented in Fig.1. A flow of noble gas leads the seeded atoms from the

\footnotetext{
* Corresponding author

Email address: simon.sels@cern.ch (S. Sels)

${ }^{1}$ Current address: CERN, CH-1211 Geneve 23, Switzerland

${ }^{2}$ Current address: GSI, D-64291 Darmstadt, Germany
}

stopping region towards the exit orifice of the cell. Originally, resonant laser ionization of the isotopes takes place inside the cell volume, where pressure and Doppler broadening effects reduce the spectral resolution and restrict a detailed knowledge of the ground-state properties [7, 9].

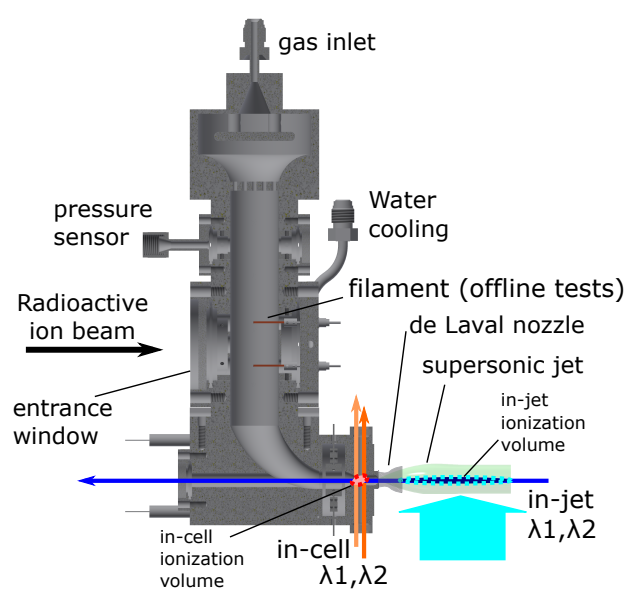

Figure 1: Drawing of the gas cell with the most important elements pointed out. Figure adjusted from [10].

For in-gas-jet spectroscopy, this process is very similar with the main difference being that the laser ionization region is moved from the gas cell to the supersonic gas jet formed by a convergent-divergent (de Laval) nozzle installed at the gas cell exit. When created with a suitable nozzle and under the right conditions, the supersonic 40 jets forms a homogeneous low density and low temperature region with a high directionality. This significantly reduces the Doppler and pressure broadening. The in-gasjet laser ionization spectroscopy method can combine the 
high efficiency of conventional in-source technique, with magnitude [11-13]. When the jet is formed under nonoptimal conditions, however, this can have adverse effects on the gas-jet conditions and therefore also on the laserspectroscopic resolution [14]. Using the stopping and fast 50 extraction by a purified noble gas, isotopes with half-lives above $100 \mathrm{~ms}$ and produced at a rate of only 1 atom every $10 \mathrm{~s}$ are expected to be measured with a spectral resolution of $\delta \nu / \nu \approx 1 \times 10^{7}(\approx 100 \mathrm{MHz})$ [12]. This makes the technique ideal for studying the heavy-element region 55 with high precision and efficiency at future radioactive ionbeam facilities such as e.g. $S^{3}$ at SPIRAL $2[15,16]$. Since stopping and extraction of isotopes is quasi element independent, the refractory elements, which are currently difficult to study with high-resolution spectroscopy at thick-

60 target ISOL facilities $[17,18]$, can also be studied using this technique. These elements are for instance of particular interest for studying the $N=Z$ nuclei.

\section{IGLIS setup}

At KU Leuven, a new laboratory has been constructed 65 with the main goal of developing the IGLIS technique and in particular the novel in-gas-jet laser ionization and spectroscopy method. The layout of the laboratory, the gascell chamber design, laser system and gas-jet formation simulations and tests have been discussed in recent pub- ${ }^{100}$ 70 lications $[10,14]$. The radio-frequency quadrupole (RFQ) ion guides, discussed in this contribution, form part of the IGLIS setup. More specifically, they are installed in the frontend vacuum chamber of the jet laboratory, depicted in Fig. 2. Their main goal is to capture the laser- ${ }^{105}$ 75 ionized species from the gas cell or gas jet and to transport them efficiently, using a combination of RF and DC electric fields, from the high-pressure environment to a low-pressure acceleration region, where a fast ion beam of $40 \mathrm{keV}$ is formed.

The accelerated ion beam can be focused and steered using an electrostatic Einzel lens and steerers located in the high-voltage section of the laboratory. Beam diagnostics downstream of the lens chamber consist of a Faraday cup (FC) and micro-channel plate (MCP) detector with ${ }^{115}$

85 a phosphor screen for beam imaging. A dipole magnet, used for mass-separation, sends the ion beam towards a detection chamber located at its focal plane.

\section{Design of the RFQ ion guides}

The purpose of the RFQ ion guides is to transport the ions from near the gas-cell exit at pressures in the order of $10^{-3}$ to $10^{-1}$ mbar, towards a high-vacuum region at $10^{-7}$ to $10^{-5}$ mbar. As the pressure in the high voltage region should be better than $10^{-5}$ mbar to avoid discharges be- ${ }^{125}$ tween HV platform and ground, the ion guides are split 95 in three sections with two $11 \mathrm{~mm}$ diameter differential

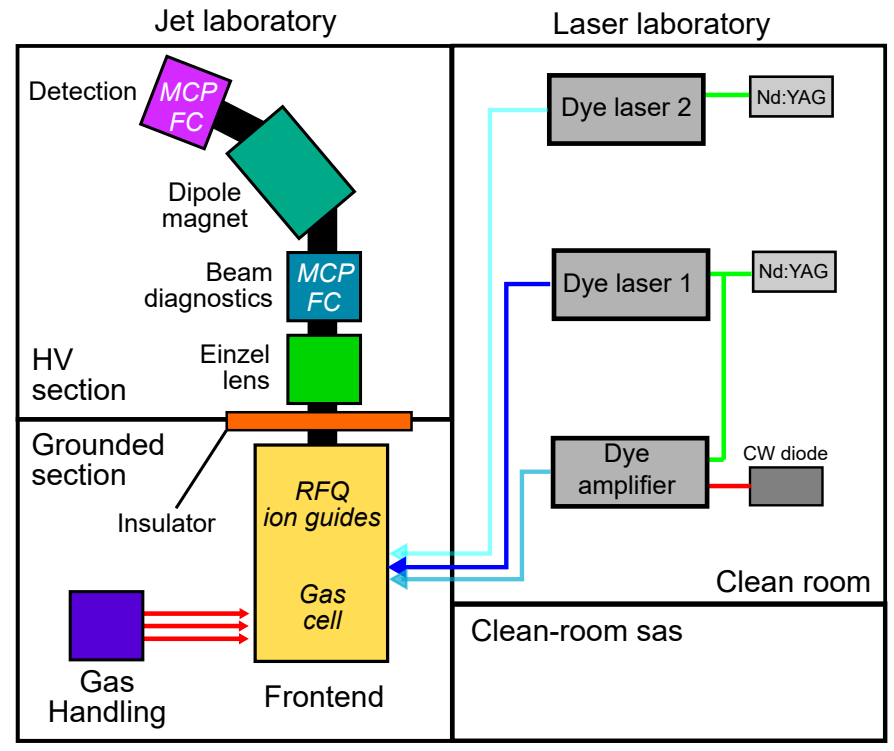

Figure 2: Schematic layout of the IGLIS laboratory at KU Leuven, comprising of the laser laboratory and the jet laboratory. More details are given in the text.

pumping sections, based on calculations of conductance of mass flow and pumping speed. The layout of the RFQ ion guides with several laser beam path options and a photograph of the frontend setup is shown in Fig. 3. The three parts of the RFQ ion guides are an S-shape RFQ (S-RFQ), a small differential-pumping RFQ (DP-RFQ) and a long ion-guide RFQ (IG-RFQ). The ion guides are driven at $1 \mathrm{MHz}$ RF frequency and the phase of all three RF generators (TTI TG2511 function generators), one for each RFQ structure are locked to one another. The generators have a maximum peak-to-peak output voltage of $10 \mathrm{~V}$. The amplitude of the RF applied to the RFQ structures is regulated by the output voltage of these generators. The $\mathrm{RF}$ voltages are passed on to an RF amplifier (HLA150 plus HF linear amplifier) with a maximum output power of $80 \mathrm{~W}$. This voltage is sent through a passive standingwave-ratio meter that indicates the ratio of outgoing versus reflected power, providing thus a measurement of how well the impedances of the RF amplifier and the RFQs acting as load are matched. Typical RF powers going into the S-RFQ were less than $1 \mathrm{~W}$. The amplified RF signals are fed into a homemade impedance-matched resonance circuit described in [19]. By a software program, the RF voltage amplitudes can be scanned for all RFQs with respect to the signal recorded in the Faraday cup to find the $\mathrm{RF}$ amplitudes for maximal transmission [20]. The layout of the RFQ ion guides and a photograph of the fronted setup is shown in Fig. 3.

\subsection{S-shape $R F Q$}

When working with a well-collimated supersonic gas jet, it is important to avoid that the gas jet enters directly the differential pumping diaphragm separating the 


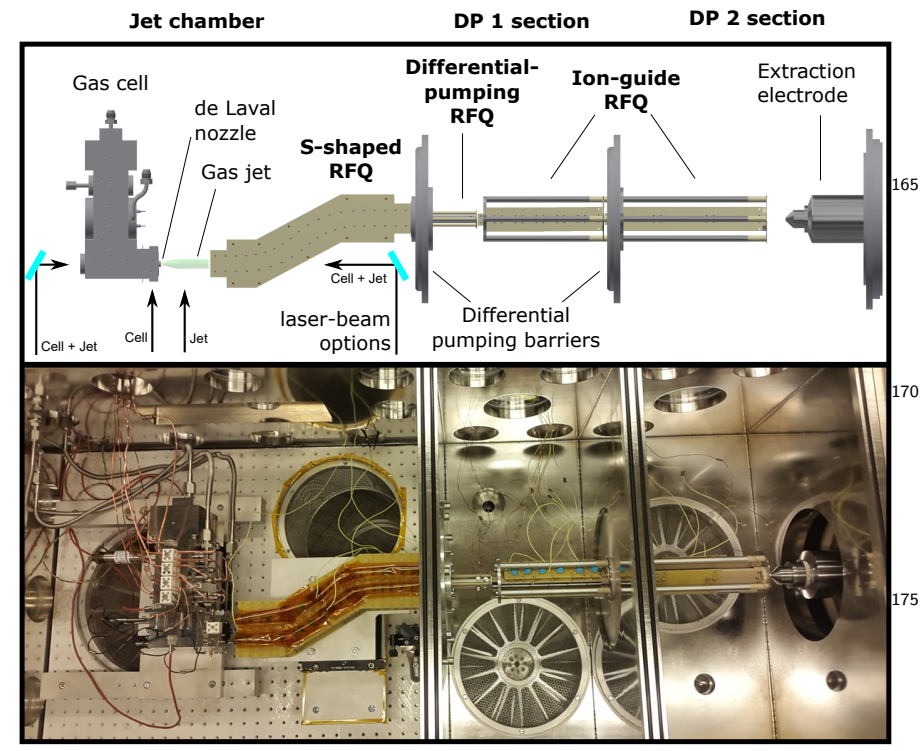

Figure 3: Top: Representation of the IGLIS frontend containing the 180 gas cell and RFQ ion guide system. Bottom: Photograph of the installed setup.

first two chambers. By bending the RFQ in an S-shape, aphragm, thus increasing the pressure suppression factor ${ }^{18}$ between the jet and the adjacent chamber. The S-bend is designed such that it is made up of two soft $45^{\circ}$ bends with a bending radius of $130 \mathrm{~mm}$, each bending in oppoDection. Both the input and output segments of the S-RFQ are straight sections for easier injection and ex-190 traction from the ion guide. The magnitude of the bent section also allows the positioning of a mirror to send a laser beam in counter-propagating direction to the gas jet.

${ }_{140}$ At the position where the laser beam passes through the RFQ, the electrodes have been shaped to allow the laser beam to interact with the full gas jet as is required for ${ }^{195}$ optimal laser ionization efficiency. The RFQ rods are split in 20 segments to which an axial DC potential gradient 145 can be applied to guide the ions along the RFQ's longitudinal axis. When considering the size of the S-RFQ, the gas jet plays an important role. In order to not disturb ${ }^{200}$ the gas-jet formation, the RFQ inner radius was chosen to accept high-Mach-number jets of up to $M=15$, i.e. jets with a $15 \mathrm{~mm}$ diameter [11] can enter the RFQ. Therefore, the inner radius $r_{0}$ of the $\mathrm{S}-\mathrm{RFQ}$, i.e. the radius of the circle inscribing the rods, was chosen to be $9 \mathrm{~mm} .^{205}$ This is intended to reduce turbulence and, as such, mitigate the potential reduction of spectral resolution in laser spectroscopy experiments.

\subsection{Differential-pumping $R F Q$}

The DP-RFQ is designed to enhanced the differential pumping and therefore resembles a small, tubular section.
LEBIT setup in Michigan State University and presented in $[21,22]$. The rods are cut in such a way that they take up minimal volume, but still produce a well-defined confining quadrupole potential. the axial electric field gradient is provided by applying a DC voltage to the two wedgeshaped electrodes. These wedges are cut, such that when combined, they form a tube surrounding the RF-carrying rods. The radius of the RFQ rods was chosen based on the results of ion-trajectory simulation performed using the SIMION software package [23] in order to optimize the ion transmission while keeping a minimal size and a maximum differential pumping, with a experimentally measured pressure suppression factor of $10^{3}$. The final inner radius of the DP-RFQ was chosen as $r_{0}=3 \mathrm{~mm}$ resulting in a near $90 \%$ ion transmission. In order to accommodate a smoother transition of the ions from the S-RFQ to the DP-RFQ, the rods of the latter extrude through the pumping diaphragm, entering the S-RFQ structure to a depth of $3 \mathrm{~mm}$.

\subsection{Ion-guide $R F Q$}

The last of the three RFQ structures has to guide the ions from the exit of the DP-RFQ up to the high-voltage extraction electrode. During this transport, the ions cross another differential pumping stage separating the DP-1 and DP-2 chambers that consists of a simple orifice with a diameter of $11 \mathrm{~mm}$. This RFQ has an inner diameter of $r_{0}=4 \mathrm{~mm}$ and is split in two by the pumping diaphragm. The final section of the RFQ is separated only $15 \mathrm{~mm}$ from the extraction electrode which is kept at a voltage of $2000 \mathrm{~V}$. In order to minimize the penetration of the HV field from the electrode into the IG-RFQ, a shielding plate is placed as final electrode, on which only a DC voltage is applied.

\subsection{Simulations}

The geometry and dimension of the electrodes important in the design phase as well as the complete performance of the RFQ ion guides was simulated using two software packages: SIMION [23] and IonCool [24]. The main difference between both packages lies in the option of modeling the ion-buffer gas interaction. While the former implements hard-sphere (HS), viscous-drag, or statistical diffusion models as ion-buffer gas interaction [25], the latter allows the use of a so-called 'realistic interaction potential' based on the $(\mathrm{n}, 4,6)$ interaction parametrization $[24,26,27]$. As the commissioning tests of the setup were performed using copper ions as test case, all simulations correspond to ${ }^{65} \mathrm{Cu}^{+}$ions moving through argon buffer gas. The output of computational fluid dynamics simulaconditions of the ion ensemble. Atomic and ionic radii needed for the ion-buffer gas drag-force and hard-sphere models were obtained from [28]. The $\mathrm{Cu}^{+}-\mathrm{Ar}$ interaction potential from [29] was used as an input for the realistic potential model calculations. 


\section{Commissioning tests}

In the first characterization tests, the transport efficiency of the RFQ ion guide system was tested. A copper filament inside the gas cell was resistively heated. The evaporated copper atoms followed the argon buffer gas flow towards the gas-cell exit, where they were resonantly ionized within the gas cell using a two-step ionization scheme as shown in [11]. The flow of argon and pressure inside the gas cell is regulated using flow controllers (Brooks gf series), while the pressure inside the jet chamber (and thus S-RFQ) was regulated by adjusting the radius of variable apertures to the vacuum pumps.

As indication for the laser-induced ion current originating from the gas cell, the S-RFQ rods were used as charge collectors. The current was measured using a picoammeter, floating at a potential of $-40 \mathrm{~V}$. Subsequently, the current measuring device was disconnected and the necessary voltages for optimal ion guiding were applied to the S-RFQ. By comparing this initial current with the curmeasured on the Faraday cup positioned in the beamdiagnostics chamber (Fig. 2), the total transport efficiency through the RFQ ion guides was determined. Typical laser-induced ion currents were in the order of $150 \mathrm{pA}$. The results of these measurements are shown in Fig.4, where ing different ion-neutral interaction potentials. A maximum transport efficiency of $91(7) \%$ could be reached at $\approx 0.1$ mbar pressure in the S-RFQ. For lower pressures, most of the losses occurred at the transition between the , where, according to simulations, the radius of the ion cloud becomes too large, owing to the low cooling process, to pass the differential pumping orifice.

Additionally, the time profile of in-gas-jet laser-produced and mass-separated copper ions were measured by the MCP-detector positioned downstream of the dipole magnet. The ionization pulse of the laser triggered the recording of the time spectra. These profiles are shown in Fig. 5 different axial voltage gradients across the S-RFQ.

The voltage gradient that is applied across the S-RFQ affects the ion pulse transit time through the ion guides. For in-gas-jet laser ionization, an ion signal is recorded between 0.65(4) and 4.8(2) ms after the laser pulse when the voltage gradient varies between $32 \mathrm{~V}$ and $0 \mathrm{~V}$ when the pressure in the jet chamber was $2.2 \times 10^{-2}$ mbar. When the voltage gradient is reversed, the ion signal disappears as expected. Not only the voltage gradient, but also the pressure inside the jet chamber plays an important role in the total transit time of the ions.

The experimental time profiles of in-gas-jet ionization are compared in Fig. 6 with simulations using both a hardsphere and realistic interaction potential models. The transport times through the RFQ ion guide are shown for different pressures and inside the gas-jet chamber and for

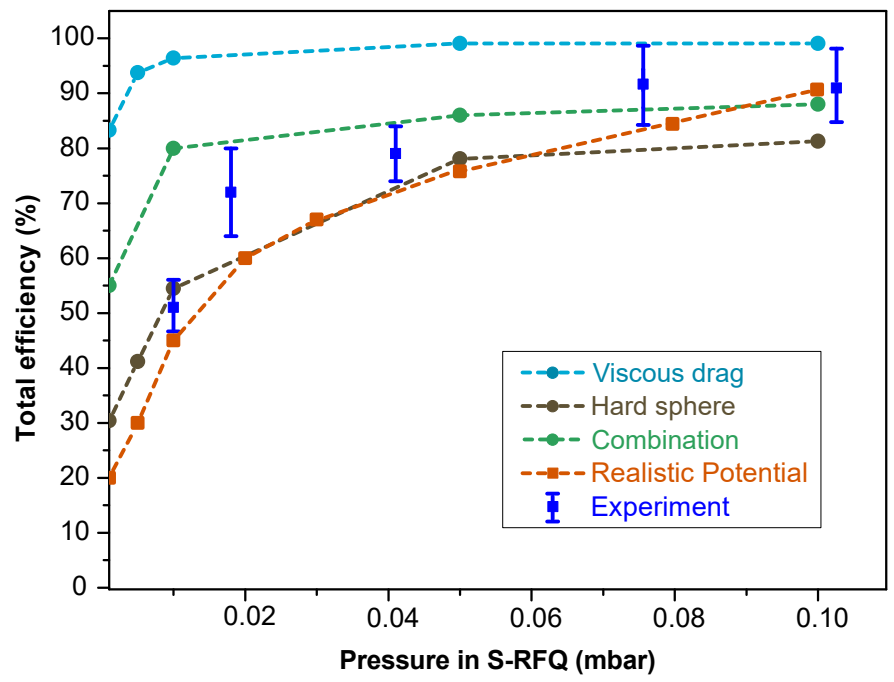

Figure 4: Comparison of experimental data for the total transport efficiency through the RFQ ion guides as a function of the pressure in the S-RFQ region with different simulations. The ion-buffer gas interaction models utilized in SIMION, include the hard-sphere, viscous-drag models and combination of both, whereas the realistic interaction potential simulations were performed with IonCool.

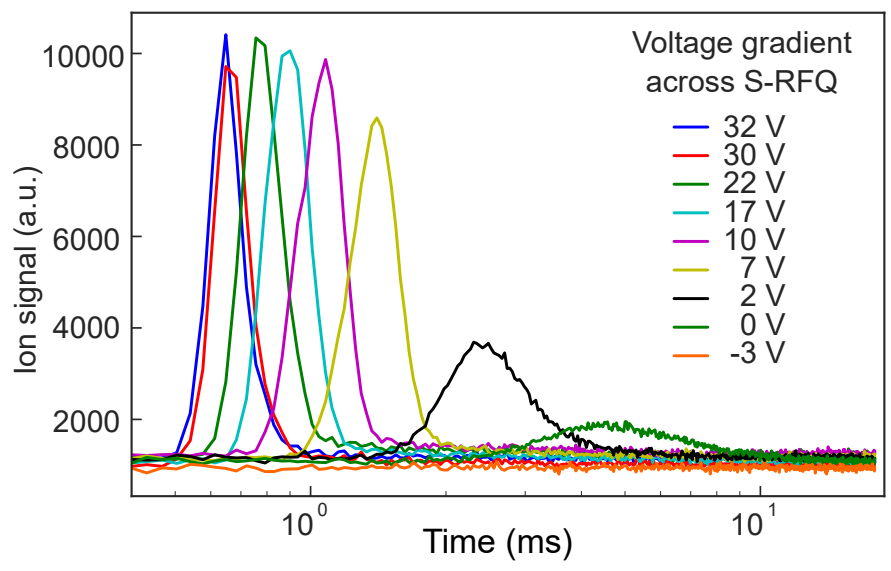

Figure 5: The ion time profiles obtained at different potentials applied across the S-RFQ for in-gas-jet laser ionization.

different DC-voltage gradients applied across the S-RFQ. While the realistic potential describes the time of arrival very well in almost all conditions, the hard-sphere model consistently underestimates the arrival time compared to the experimental values.

In order to estimate the longitudinal ion energy spread of the ion beam, the ion transport was measured as a function of the retardation bias voltage applied to the differential pumping barrier between section DP-1 and DP-2. The results are shown in Fig. 7. The data was fitted with an S-shaped function, from which the longitudinal energy spread was estimated as the full width at half maximum (FWHM) of the fit function's derivative. This resulted in an energy spread of $1.9(8) \mathrm{eV}$, which is in reasonable 


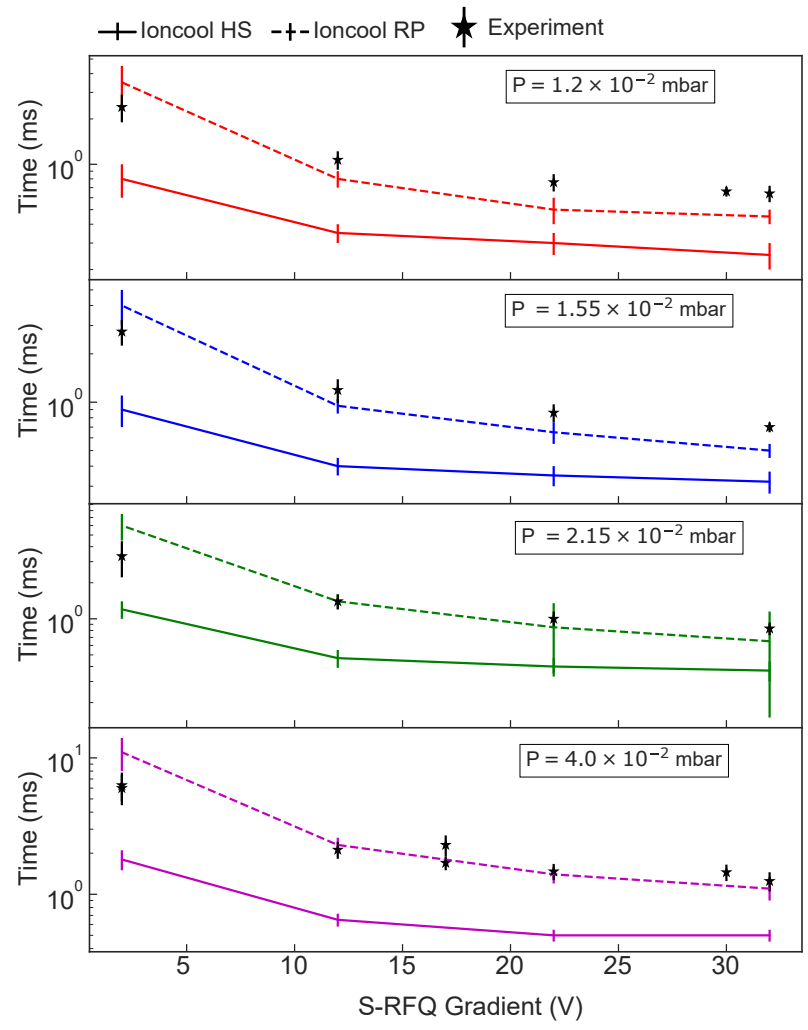

Figure 6: Comparison of experimental and calculated transport times through the RFQ ion guides using IonCool, with either the hard sphere (HS) or realistic potential (RP) models. $P$ represents the pressure in the jet chamber.

agreement with the simulated value of $0.9(5) \mathrm{eV}$ using the realistic potential of IonCool.

Preliminary measurements of the transverse emittance were performed to further characterize the ion-beam quality coming from the in-gas laser ion source and RFQs. The tests were performed using a method detailed in $[8,19,30$, 31]. A measurement of the beam width is performed using a MCP and phosphor screen combination, while varying the focusing voltage of an einzel lens. While strong aberations in the ion beam at the time of measuring hindered a clean measurement, a preliminary emittance value of $12(5) \pi \mathrm{mm}$ mrad at $2 \mathrm{keV}$ was extracted. This value agrees with IonCool simulations where the same pressure and S-RFQ voltage gradient were used as in experiment, which returned a value of $10 \pi \mathrm{mm}$ mrad.

From the transport-efficiency measurements an operational diagram can be constructed which relates the optimal RFQ working conditions for different Mach numbers of the supersonic gas-jet. Calculations of the IGLISlaboratory pumping system relate the the Mach number ${ }^{320}$ to a gas-jet chamber pressure for optimal jet formation. Such calculations were performed for various pressures inside the gas cell $P_{0}$ in [10] and are indicated by the blue, red and dashed black curve in Fig. 8. The green area in

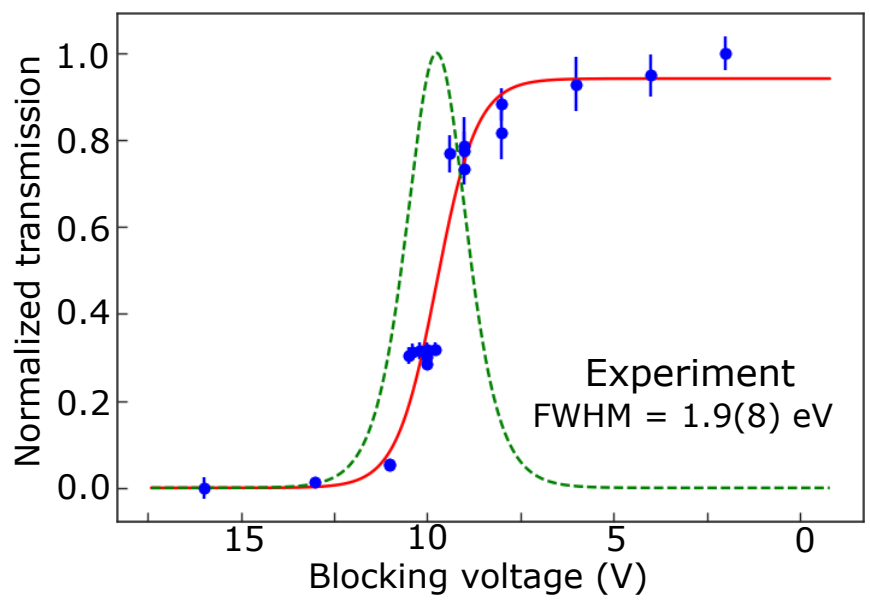

Figure 7: Measurement of the energy spread at the differential pumping barrier between DP-1 and DP-2. In green, the derivative of the red-colored fit of the data to an S-curve function is shown. The FWHM of the green function is $1.9(8) \mathrm{eV}$. This measurement was performed at a pressure of 0.1 mbar in the jet chamber.

this figure corresponds to the region for which the total transport efficiency of the RFQ ion guides is higher than $50 \%$. The upper limit in pressure is imposed by an onset of electrical discharges present in the S-RFQ at these higher pressures. This green area in Fig. 8 limits the optimal gasjet Mach numbers at which the setup can be operated.

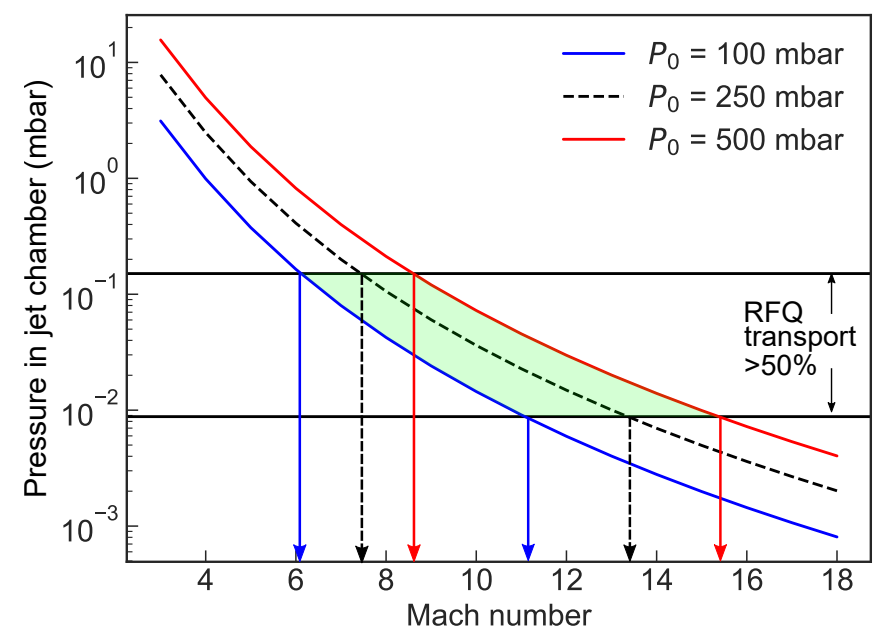

Figure 8: Operational diagram for the optimal IGLIS Mach-number working conditions.

\section{Conclusion}

RFQ ion guides, specifically designed to improve the performance of IGLIS experiments, have been designed and commissioned. The results of the commissioning experiments agree well with the expectations from simulations. In optimal conditions, the RFQ ion guide showed a 
325 maximum total transport efficiency of $91(7) \%$ and a longitudinal ion energy spread of $1.9(8) \mathrm{eV}$. The transport ${ }^{385}$ time through the RFQ ion guides for in-gas-jet ionization ranges between $0.65(4) \mathrm{ms}$ and $4.8(2) \mathrm{ms}$, but is in any case significantly shorter then the extraction time of

330 the gas cell. A preliminary transverse emittance value of ${ }^{390}$ 12(5) $\pi \mathrm{mm}$ mrad was extracted from a combination of experiment with simulations. However, new and detailed emittance measurements are planned in the near future. Based on the results from the commissioning tests, an op- ${ }^{395}$ erational diagram was constructed, indicating at which Mach-number interval the setup is expected to perform optimally. The ion guides are installed and operational in the new IGLIS laboratory at KU Leuven, where the de- ${ }^{400}$ velopment of the in-gas-jet laser ionization spectroscopy technique is ongoing.

\section{Acknowledgments}

We would like to thank S. Schwarz for kindly providing the IonCool code that he developed. This work has received funding from Research Foundation Flanders (FWO, Belgium), by GOA/2015/010 (BOF KU Leuven), the Interuniversity Attraction Poles Programme initiated by the Belgian Science Policy Office (BriX network P7/12), from the European Research Council (ERC-2011-AdG-291561HELIOS). S. S. acknowledges a SB PhD grant from the former Belgian Agency for Innovation by Science and Technology (IWT), now incorporated in FWO-Vlaanderen.

\section{References}

[1] K. Blaum, J. Dilling, and W. Nörtershäuser, "Precision atomic ${ }_{425}$ physics techniques for nuclear physics with radioactive beams," Phys. Scr., vol. T152, p. 014017, Jan 2013.

[2] P. Campbell, I. Moore, and M. Pearson, "Laser spectroscopy for nuclear structure physics," Progress in Particle and Nuclear Physics, vol. 86, pp. 127 - 180, 2016.

[3] R. Neugart et al., "Collinear laser spectroscopy at ISOLDE: new methods and highlights," Journal of Physics G: Nuclear and Particle Physics, vol. 44, no. 6, p. 064002, 2017.

[4] B. A. Marsh et al., "Characterization of the shape-staggering effect in mercury nuclei," Nature Physics, vol. 14, no. $12,_{435}$ pp. 1163-1167, 2018.

[5] S. Sels et al., "Shape staggering of mid-shell mercury isotopes from in-source laser spectroscopy compared with density functional theory and monte carlo shell model calculations," Accepted to Phys. Rev. C., 2019.

[6] H. De Witte et al., "Nuclear charge radii of neutron-deficient lead isotopes beyond $\mathrm{N}=104$ midshell investigated by in-source laser spectroscopy," Phys. Rev. Lett., vol. 98, p. 112502, Mar 2007.

[7] T. E. Cocolios, A. N. Andreyev, B. Bastin, N. Bree, J. Büscher, J. Elseviers, J. Gentens, M. Huyse, Y. Kudryavtsev, D. Pauwels, T. Sonoda, P. Van den Bergh, and P. Van Duppen, "Magnetic dipole moment of ${ }^{57,59} \mathrm{Cu}$ measured by in-gas-cell laser spectroscopy," Phys. Rev. Lett., vol. 103, p. 102501, Aug 2009.

[8] R. Ferrer, A. Kwiatkowski, G. Bollen, D. Lincoln, D. Morrissey, G. Pang, R. Ringle, J. Savory, and S. Schwarz, "Ion beam properties after mass filtering with a linear radiofrequency quadrupole," Nuclear Instruments and Methods in Physics Research Section A: Accelerators, Spectrometers, Detectors and Associated Equipment, vol. 735, pp. 382 - 389, 2014.
[9] R. Ferrer et al., "In-gas-cell laser ionization spectroscopy in the vicinity of 100Sn: Magnetic moments and mean-square charge radii of $\mathrm{N}=5054 \mathrm{ag}, "$ Physics Letters B, vol. 728, pp. $191-197$, 2014.

[10] Y. Kudryavtsev et al., "A new in-gas-laser ionization and spectroscopy laboratory for off-line studies at KU Leuven," Nuclear Instruments and Methods in Physics Research Section B: Beam Interactions with Materials and Atoms, vol. 376, no. Supplement C, pp. 345 - 352, 2016. Proceedings of the XVIIth International Conference on Electromagnetic Isotope Separators and Related Topics (EMIS2015), Grand Rapids, MI, U.S.A., 11-15 May 2015.

[11] Y. Kudryavtsev, R. Ferrer, M. Huyse, P. V. den Bergh, and P. V. Duppen, "The in-gas-jet laser ion source: Resonance ionization spectroscopy of radioactive atoms in supersonic gas jets," $\mathrm{Nu}$ clear Instruments and Methods in Physics Research Section B: Beam Interactions with Materials and Atoms, vol. 297, pp. 7 $22,2013$.

[12] R. Ferrer et al., "Towards high-resolution laser ionization spectroscopy of the heaviest elements in supersonic gas jet expansion," Nature Communications, vol. 8, pp. 14520 EP -, Feb 2017. Article.

[13] C. Granados et al., "In-gas laser ionization and spectroscopy of actinium isotopes near the $\mathrm{N}=126$ closed shell," Phys. Rev. C, vol. 96, p. 054331, Nov 2017.

[14] A. Zadvornaya et al., "Characterization of supersonic gas jets for high-resolution laser ionization spectroscopy of heavy elements," Phys. Rev. X, vol. 8, p. 041008, Oct 2018.

[15] R. Ferrer et al., "In gas laser ionization and spectroscopy experiments at the superconducting separator spectrometer (S3): Conceptual studies and preliminary design," Nuclear Instruments and Methods in Physics Research Section B: Beam Interactions with Materials and Atoms, vol. 317, pp. 570 - 581, 2013. XVIth International Conference on ElectroMagnetic Isotope Separators and Techniques Related to their Applications, December 27, 2012 at Matsue, Japan.

[16] F. Déchery et al., "The super separator spectrometer S3 and the associated detection systems: Sirius \& LEB-REGLIS3," $\mathrm{Nu}$ clear Instruments and Methods in Physics Research Section B: Beam Interactions with Materials and Atoms, vol. 376, pp. 125 - 130, 2016. Proceedings of the XVIIth International Conference on Electromagnetic Isotope Separators and Related Topics (EMIS2015), Grand Rapids, MI, U.S.A., 11-15 May 2015.

[17] U. Köster et al., "Intense radioactive-ion beams produced with the isol method," Eur Phys J A, vol. 15, pp. 255-263, 2002.

[18] A. Kronenberg et al., "Molecular sidebands of refractory elements for isol," Nuclear Instruments and Methods in Physics Research Section B: Beam Interactions with Materials and Atoms, vol. 266, no. 19, pp. 4252 - 4256, 2008. Proceedings of the XVth International Conference on Electromagnetic Isotope Separators and Techniques Related to their Applications.

[19] S. Sels, "Laser spectroscopy of neutron-deficient mercury isotopes and commissioning of a gas-jet based RFQ ion guide, PhD thesis," 2018.

[20] K. Dockx et al., "A new control system for high precision in-gas laser ionization and spectroscopy experiments at KU Leuven," Submitted to NIM B as proceedings to the EMIS 2018 conference, 2019.

[21] S. Schwarz et al., "A second-generation ion beam buncher and cooler," Nuclear Instruments and Methods in Physics Research Section B: Beam Interactions with Materials and Atoms, vol. 204, pp. $474-477$, 2003. 14th International Conference on Electromagnetic Isotope Separators and Techniques Related to their Applications.

[22] G. Bollen et al., "Beam cooling at the low-energy-beam and iontrap facility at nscl/msu," Nuclear Instruments and Methods in Physics Research Section A: Accelerators, Spectrometers, Detectors and Associated Equipment, vol. 532, no. 1, pp. 203 209, 2004. International Workshop on Beam Cooling and Related Topics.

[23] D. Manura and D. Dahl, "SIMION (r) 8.0 user manual," Jan- 
uary 2008.

[24] S. Schwarz, "Ioncool: A versatile code to characterize gas-filled ion bunchers and coolers (not only) for nuclear physics applications," Nuclear Instruments and Methods in Physics Research Section A: Accelerators, Spectrometers, Detectors and Associated Equipment, vol. 566, no. 2, pp. 233 - 243, 2006.

[25] D. Manura, "Notes on the SIMION HS1 collision model," 2007.

[26] A. Kellerbauer, T. Kim, R. Moore, and P. Varfalvy, "Buffer gas cooling of ion beams," Nuclear Instruments and Methods in Physics Research Section A: Accelerators, Spectrometers, Detectors and Associated Equipment, vol. 469, no. 2, pp. $276-$ $285,2001$.

[27] S. Schwarz, "Simulations for ion traps buffer gas cooling," in The Euroschool Lectures on Physics with Exotic Beams, Vol. $I$ (K. Blaum and F. Herfurt, eds.), Lecture Notes in Physics, pp. $97-116$, Springer Berlin Heidelberg, 2008.

[28] "Webelements database, https://www.webelements.com/."

[29] X. Tong et al., "Interactions of $\mathrm{MzX}$ complexes $(\mathrm{M}=\mathrm{Cu}, \mathrm{Ag}$, and $\mathrm{Au} ; \mathrm{X}=\mathrm{He}, \mathrm{Ne}$, and $\mathrm{Ar}$; and $\mathrm{z}=1)$," The Journal of Chemical Physics, vol. 134, no. 2, p. 024306, 2011.

[30] K. T. McDonald and D. P. Russell, "Methods of emittance measurement," Frontiers of Particle Beams; Observation, Diagnosis and Correction, pp. 122-132, 1989.

[31] B. Barquest, "Phd thesis, michigan state university: An advanced ion guide for beam cooling and bunching for collinear 